\title{
FIELD MEASUREMENTS ON THE HERA E-RING MAGNETS.
}

O.Kaul, Y.Holler, K.Sinram, U.Berghaus

Deutsches Elektronen Synchrotron DESY, Notkestr. 85, 2000 Hamburg 52, W.Germany

Summary: In the HERA electron storage ring and its injection channels, a total of 454 dipoles, 716 quadrupoles and 420 sextupoles of various types, will be installed. The pole profiles of all magnet types have been designed with the aid of a computer program [ 1 ]. This contribution covers the details of the devices developed to measure the fields of these magnets as well as the results of the measurements. All magnets are measured using the moving coil technique. The coil length is chosen considerably longer than the iron yoke to include fringe fields and to get the integrated values of the main and higher order poles. For the field measurements of the different magnet types, 5 individual test benches have been developed.

\section{Definitions}

Since the period of the betatron oscillations exceeds the length of the magnet by far, integrated measurements will adequately determine the characteristics of the magnets, provided that the fringe fields are included. In the field region of a beam transport magnet, where the current density is zero, the magnetic field distribution may be described by a two-dimensional scalar potential $V(r, \theta)$, which satifies the Laplace equation.

(1) $\nabla^{2} V(r, \theta)=0$

The general solution for a m-pole magnet may be written as

(2) $V(r, \theta)=\int_{n}^{A} A(2 n-1) m / 2 \sin ((2 n-1) m / 2 \theta) r^{(2 n-1)}$ where $A(2 n-1) m / 2$ are the so-called harmonic coefficients. The magnetic induction is then given by

(3) $\vec{B}=-\mu_{0} \vec{\nabla} V(r, \theta)$

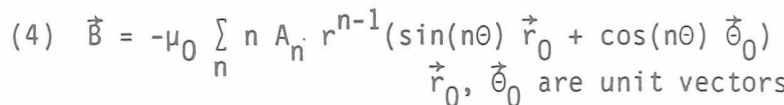

\section{Multipole Measuring System}

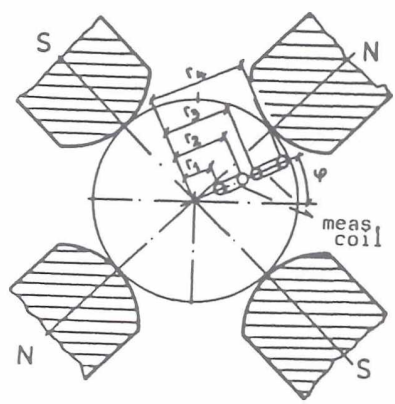

Fig. 1: Rotating coil geometry

$$
\text { (5) } \begin{aligned}
& E_{\Theta, n}=\mu_{0} N_{1} n w A_{n} \\
&\left(r_{1}^{n}-r_{2}^{n}\right) \sin (n w t)
\end{aligned}
$$

Fig. 1 depicts two measuring coils rotating with constant angular velocity $w$ around the symmetry axis of a quadrupole picking up the azimuthal field component. They are actuated by stepping motors. A high resolution encoder measures the actual angle of rotation.

The voltage induced in the coil by the $n$-th harmonic is

The voltage originating during one complete revolution is digitally recorded at equidistant angular steps. The voltage values undergo a discrete Fourier Transformation yielding the desired multipole coefficients.

\subsection{Main Field Compensation}

The quality of a magnet is judged by the magnitude of unwanted field components which may be of higher or lower order than the desired pole. Due to the fact that these contributions are generally very small, one suppresses the main component in the field measurement and thereby increases the sensitivity to the remaining harmonics. We employ a system of two coils electrically connected in antiseries (Fig. 1). Total suppression of the $n$-th order multipole can be achieved by choosing the turn ratio and radii according to

(6) $N_{2}\left(r_{4}^{n}-r_{3}^{n}\right)=N_{1}\left(r_{2}^{n}-r_{1}^{n}\right)$

Although the other harmonics are more or less attenuated by this technique, they can be accurately determined after appropriate amplification. Mandatory conditions are a good stability of the rod carrying the measuring coil and the absence of vibrations in the driving mechanism.

Fig. 2 shows the general layout of the system.

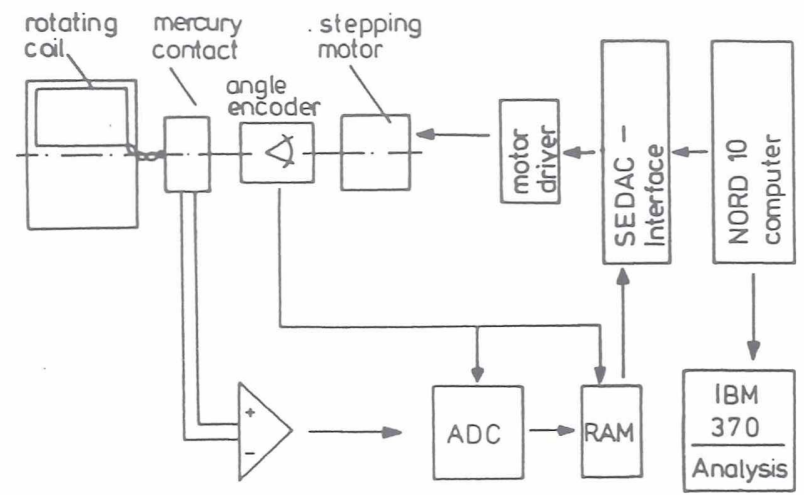

Fig. 2: General layout of the system

\section{Dipole Measurement System}

Two seperate test benches have been constructed for the HERA main dipoles type BE as well as for the dipoles installed in the injection channels.

\subsection{HERA Dipole BE}

\subsubsection{Field Homogeneity}

For all dipole magnets the integral multipole components are measured-with a horizontally moving coil. Fig. 3 outlines the measuring method for the $B E$ dipole.

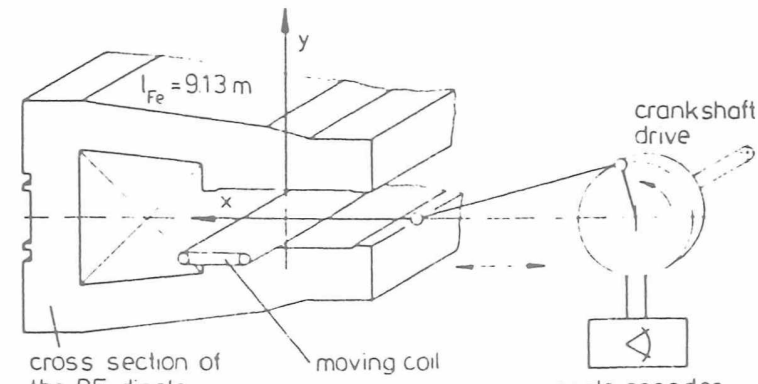

the BE dipole

angle encoder

Fig. 3: Horizontally moving coil inside the BE dipole

An eccentric drive sweeps the coil in the magnet's plane of symmetry with a radial stroke of $+/-45 \mathrm{~mm}$. During one full rotation of the stepping motor 
( $T=2 \mathrm{sec}$.), the 13 bit angle encoder triggers 8192 separate conversions of the induced voltage with a resolution of 12 bits. Because the measurement takes place just in one plane, the Fourier expansion (4) of the magnetic induction $B$ is reduced to a polynomial in $x$.

$$
\text { (7) } B_{n}=A_{n} x^{n-1}
$$

The induced voltage, normalized by the velocity is given by

$$
\text { (8) } \begin{aligned}
U_{\text {ind. }}(x) / v_{1 \text { in. }}(x)= & B(x)-B(x+d x) \\
& d x \text { : coil width }
\end{aligned}
$$

After a simple transformation, the coefficients of the magnetic field are extracted from a polynomial fit of the data. The dipole strength cannot be measured with this technique. This is because the induced voltage is zero, as can be seen from (8), in the case of a constant magnetic field. Fig. 4a,b represent typical results of a $B E$ dipole, running at an exciting current of $6778 \mathrm{~A}$. The magnetic field in the gap at this current is $0.163 \mathrm{~T}$, corresponding to a beam energy of 30 $\mathrm{GeV}$. Because the quadrupole and sextupole components $(n=2,3)$ of the bending magnet can be compensated with the quadrupole and sextupole magnets installed in the normal HERA-cell the radial pole contour has been shaped to minimize the field deviations after subtraction of the $(n=2,3)$ components.

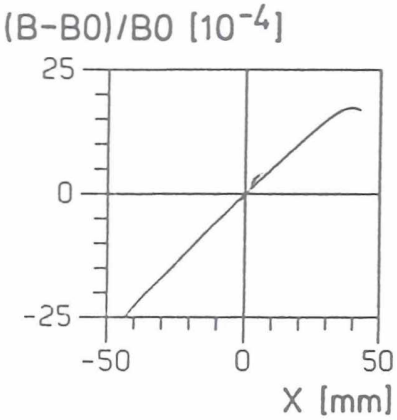

(a)

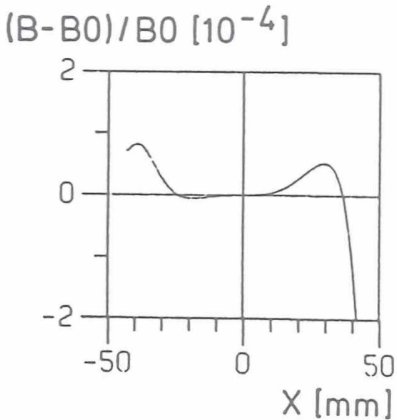

(b)
Fig. 4: Field distributions: a) total, b) after substraction of the $n=2,3$ components

\subsubsection{Dipole Strength}

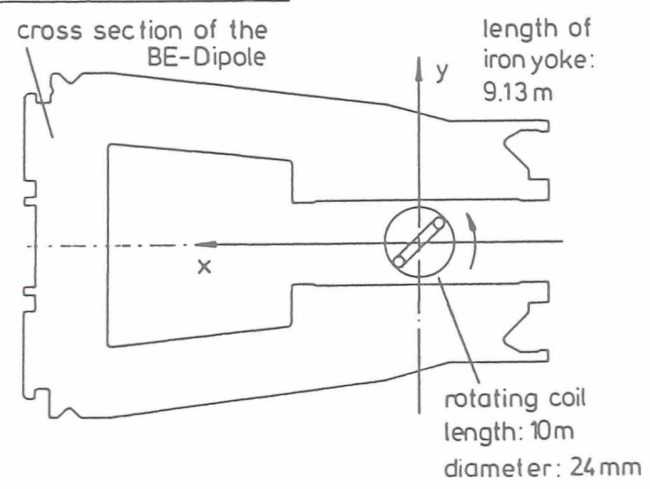

Fig. 5: Principle of $\int B d l$ measurement

Fig. 5 displays the principle of this measurement. The rotating coil $(1=10 \mathrm{~m})$, is driven at the ends by stepping motors with a rotating velocity of 0.75 $\mathrm{Hz}$. As above, the angle encoder triggers 8192 separate measurements per rotation. To increase the accuracy and the long term stability of the results, the measuring coil is connected in antiseries with an identical coil rotating in a reference magnet of the same type. Since the same exciting current passes through both magnets and the reference magnet and the current itself are monitored independently, the dipole strength of the sample magnet in relation to the reference magnet can be determined to an accuracy of +/$1.5 \times 10^{-4}$.

\subsection{Injection Dipoles PR and EL}

\subsubsection{Field Homogeneity}

The field homogeneity of the PR (proton) and EL (electron) dipoles is measured with horizontally moving coils of $6 \mathrm{~m}(P R)$ and $3 \mathrm{~m}$ (EL) length. The cross section of these dipoles (window frame type) allow an attachment of the coils solely at the ends. An air cushion bearing totally eliminates the friction of the coils and greatly reduces the excitation of mechanical oscillations (Fig. 6).

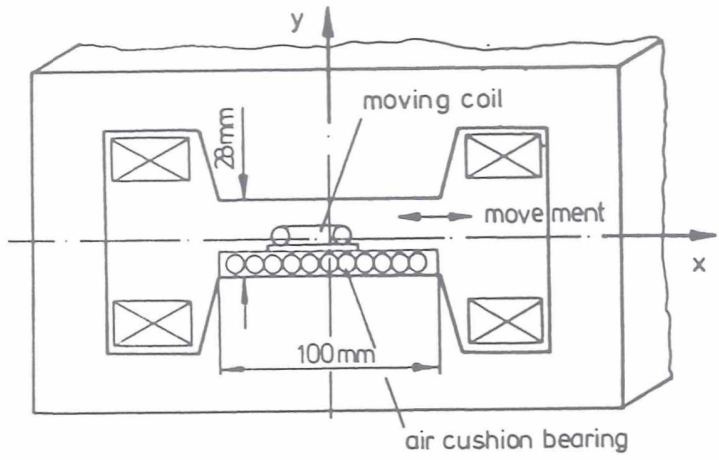

Fig. 6: Injection dipole: horizontally moving coil hovering on an air cushion bearing

$(B-B O) / B O\left[10^{-4}\right]$

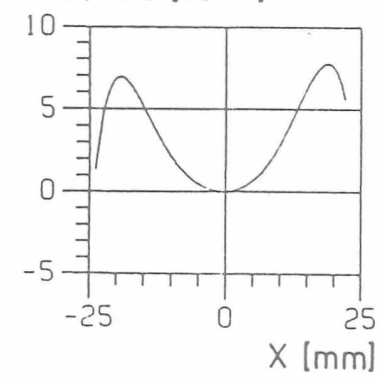

Fig. 7: Magnetic field distribution at $B_{0}=1.82 \mathrm{~T}$

A typical field distribution recorded with such a coil is shown in Fig. 7. The measurement was carried out at a field strength of 1.82. Tesla corresponding to the maximum injection energy of $40 \mathrm{GeV}$ protons (PR dipole).

\subsubsection{Dipole Strength}

For these measurements the same coil as above was used resting in the center of the magnet while the exciting current was ramped up and down. The time integral of the induced voltage is directly proportional to the $\int\left(\begin{array}{ll}B & d l\end{array}\right)$ to be determined. The integration is performed with a VFC* (Voltage to Frequency Converter) feeding a counter. We have

$$
\begin{array}{ll}
\text { (9) }-N W\left(\int B d l-\int B_{\text {rem }} d l\right)=\int u d t=C \text { Counts } \\
N: \text { Number of coil windings } W: \text { Coil width } \\
B_{\text {rem }}: \text { Remanence } & u: \text { Voltage } \\
C: \text { VFC constant } &
\end{array}
$$

The portion of the $\int B d l$ originating from the remanent field in the yoke iron is measured with a Hall probe.

* HP 2212A 


\section{Results of the Quadrupole and Sextupole Tests}

A typical measurement of a $Q E$ quadrupole is shown in Fig. 8.

$\mathrm{B}_{\mathrm{n}} / \mathrm{B}_{2}$

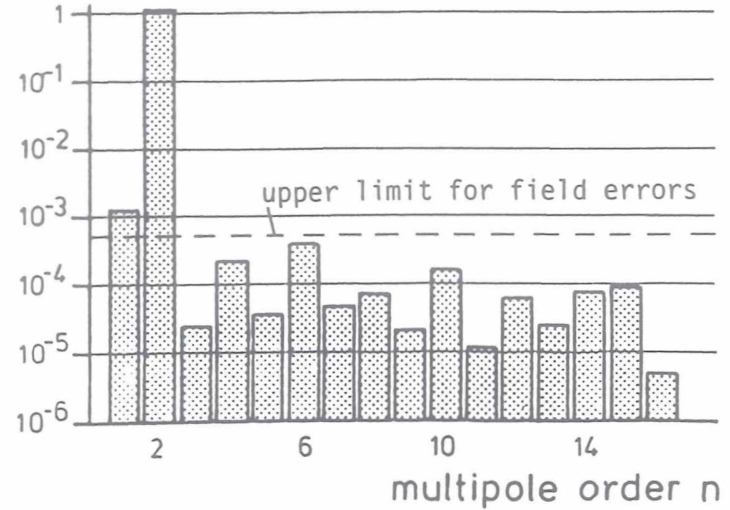

Fig. 8: Multipole distribution for a QE quadrupole

The data were taken at a current of $400 \mathrm{~A}$ corresponding to a beam energy of $30 \mathrm{GeV}$. The plotted field components are converted to the bore radius of $37 \mathrm{~mm}$ and normalized to the design value of the main pole. All higher order components are well below the $5 \times 10^{-4}$ upper limit imposed by theoretical beam quality considerations ( $E=35 \mathrm{GeV}, 60$ deg optics). This holds for all 272 specimens and includes the prominent (due to the quadrupole symmetry so-called allowed) multipoles with $n=6,10,14$. The dipole component can mainly be attributed to inaccurate adjustments of the measuring coil $\left(B_{(n=1)}\right.$ rel $=2 \times 10^{-3} \Leftrightarrow 0.07 \mathrm{~mm}$ misalignment). Fig. 9 shows the distribution of the normalized quadrupole strengths of 272 tested magnets.

N

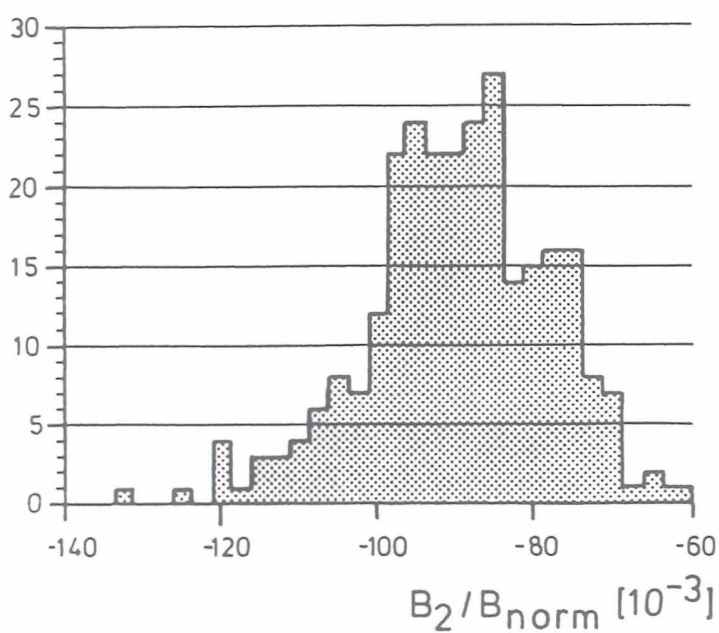

Fig. 9: Strengths of all tested $Q E$ quads.

The standard deviation is $0.12 \%$ and exceeds the beam optics requirements of $0.1 \%$ by a small amount. For this reason the magnets are subdivided into two classes with correspondingly smaller standard deviations.

Fig. 10 shows the multipole distribution for the SK sextupole.

The field errors are within the tolerance levels, because as in the case of the quadrupoles the $n=1,2$ components are mainly due to misalignments of the mea- suring coil.

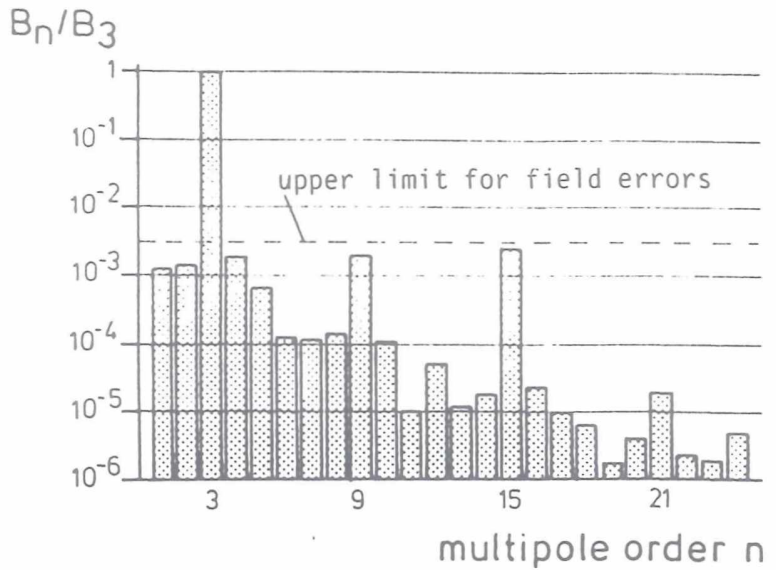

Fig. 10: Multipole distribution for a SK sextupole

\section{Results of the Dipole Tests}

\subsection{HERA e-Dipole}

The field deviations in the radial interval of $-40 \mathrm{~mm}<x<40 \mathrm{~mm}$ do not exceed $+/-1.5 \times 10^{-4} B(x=0)$ after subtraction of the $(n=2,3)$-components for a total of 170 dipoles tested so far. The gap heights however varied in the course of the series production (Fig. 1la) affecting the quad term (Fig. 11b) as well as the dipole strength (Fig. 11C).

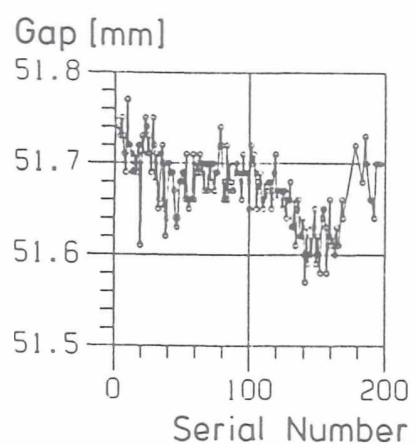

(a)

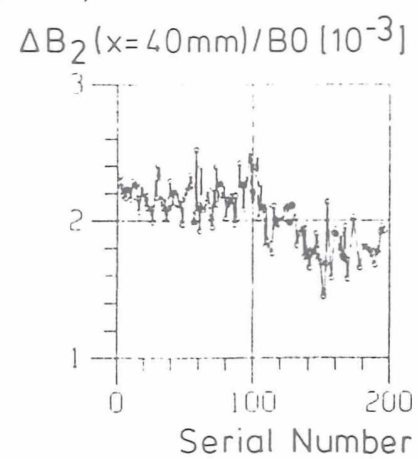

(b)

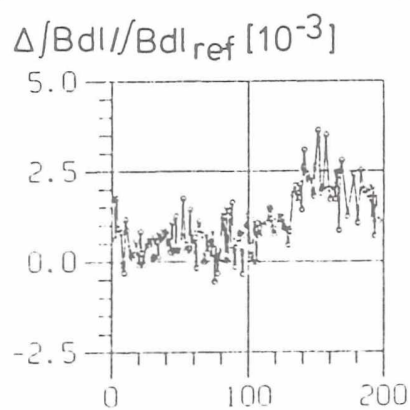

Serial Number

(c)

Fig. 11: BE dipoles: a) development of gapheight, b) quadrupole component, c) integrated magnetic strength

These interdependences $c$ an be estimated by $\Delta$ gapheight $=0.1 \mathrm{~mm} \Leftrightarrow \Delta B_{2}(x=40 \mathrm{~mm}) / B(x=0)=5 \times 10^{-4}$

$$
\Leftrightarrow \quad \Delta \int B d l=2 \times 10^{-3}
$$


A scheme for sorting the measured dipoles placing them in the ring is employed to nearly neutralize these effects. The spread of the dipole strengths determined by measurements relative to the reference magnet is depicted in Fig. 12 for a beam energy of $14 \mathrm{GeV}$.

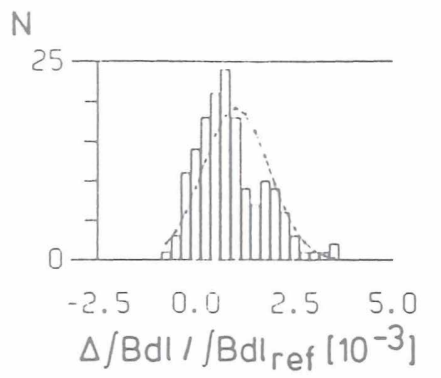

Fig. 12: Distribution of the integrated magnetic strengths

The results for 14,30 and $50 \mathrm{GeV}$ respectively are summarized in Table 1 . The comparatively strong variations at $E=50 \mathrm{GeV}$ will be smoothed by means of one correction dipole for every two main dipoles. Their maximum deflecting strength is $0.045 \mathrm{Tm}$ corresponding to $1.8 \%$ of the main dipole's strength.

\subsection{Injection Dipoles}

In the $P R$ and $E L$

$(\mathrm{B}-\mathrm{BO}) / \mathrm{BO}\left[10^{-4}\right]$

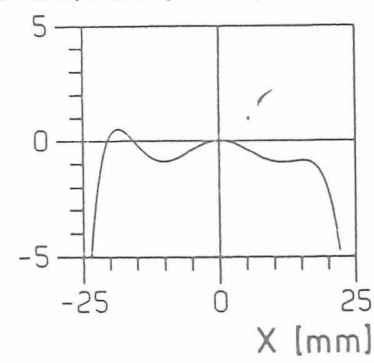

Fig. 13: Magnetic field distribution after shimming dipole magnets additional longitudinal shim strips had to be glued to the center line of the pole faces in order to reduce the field errors from $8 \times$ $10^{-4}$ to $\pm 1 \times 10^{-4}$ (Fig. 13). The dimensions of these shims $(1000 \times 20 \times$ $0.1 \mathrm{~mm}$ and $5000 \times 20 \times$ $0.1 \mathrm{~mm}$ for the PR and EL magnets respectively) were optimized for the injection energies of 40 $\mathrm{GeV}$ (PR) and $14 \mathrm{GeV}$ (EL) respectively. With decreasing energy and hence less saturation effects, the relative field errors $(B-B(0)) / B(0)$ along the radial coordinate are increasing and rise to a maximum of $12 \times 10^{-4}$ at the boundaries of the usable aperture of $+/-16 \mathrm{~mm}$. The distributions of the dipole strength of all PR and EL magnets can be seen in Fig. 14, supplemented by the final results in table 1 .
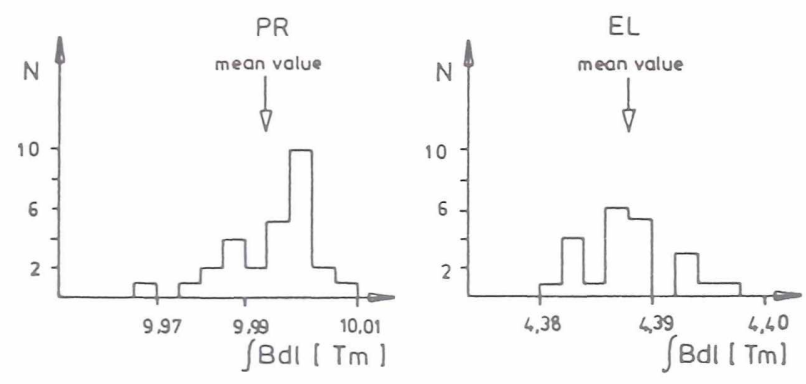

Fig. 14: Injection dipoles: integrated magnetic strengths

\begin{tabular}{|c|c|c|c|c|}
\hline $\begin{array}{l}\text { dipole } \\
\text { typ }\end{array}$ & $\begin{array}{l}\text { number } \\
\text { of } \\
\text { dipoles }\end{array}$ & $\begin{array}{c}E \\
{[\mathrm{GeV}]}\end{array}$ & $\begin{array}{l}\int B d l \\
{[\mathrm{Tm}]}\end{array}$ & $\begin{array}{c}\sigma\left(\int B d l / \int B d l_{r e f}\right) \\
{\left[10^{-3}\right]}\end{array}$ \\
\hline \multirow{3}{*}{$\begin{array}{l}\text { HERA } \\
\mathrm{e}^{-} \\
\mathrm{BE}\end{array}$} & \multirow{3}{*}{170} & 14 & 0.7038 & 0.82 \\
\hline & & 30 & 1.503 & 1.05 \\
\hline & & 50 & 2.480 & 1.35 \\
\hline PR & 28 & 40 & 9.996 & 0.77 \\
\hline EL & 22 & 14 & 4.386 & 0.92 \\
\hline
\end{tabular}

Table: Results of the dipole tests

\section{Conclusions}

On the basis of the statistical data extracted from the measurements carried out so far, we can state, that the magnets to be installed in the $e^{-}$-ring and injection channels of HERA will clearly meet the projected quality standards. The measuring machines are well suited with respect to accuracy and ease of use for the extensive test programs already done or still lying ahead of us.

\section{References}

[ 1 ] Ch. Iselin, CERN, Computer Program Library, T 600 (1971) 